\title{
Rapid Development of Location-based Apps: Saving Lives during a Pandemic - the South Korean Experience
}

\author{
Bola Michelle Ju, Lesley Arnold and Kathrine Kelm
}

The first confirmed case of COVID-19 in South Korea was recorded in January 2020. The government took swift wide-ranging measures to protect the health and wellbeing of citizens using geospatial data and information communications technology, which included immediate upgrades to the Emergency Broadcast System. Several location-based applications were developed during the height of the evolving pandemic. The speed with which these applications were developed and subsequently used by the community was a remarkable feat, and one that has set a benchmark for other countries aiming to flatten the curve of infections. These Apps have delivered benefits contributing to the prevention of community transmissions, particularly from the influx of overseas travellers. The Smart City Data Hub system enhanced resiliency during the second wave in Daegu where mass infections eventuated at a large religious gathering. The system enabled the analysis of pathway tracings of people who attended the event using big data analytics and data provided by credit card, transport and mobile companies. This chapter describes seven major location-based applications. It discusses the mechanisms behind each system, the technological and data foundations that enabled their development and operation, and how they are contributing to strengthening resiliency efforts during the COVID-19 crisis. The chapter also describes how the South Korea Government has previously made use of geospatial information and data processing systems to respond to past pandemics, and how the lessons learned from these earlier developments has contributed to the success of COVID-19 response efforts today. The major technology, data, policy and institutional arrangements that enabled the COVID-19 applications to be developed in such a short space of time are discussed, including broad direction for future research and development opportunities to manage future pandemics.

\subsection{Introduction}

Global pandemics have occurred with devastating impact over the past centuries. The well-known Black Death outbreak in the 14th century killed around 50 million lives [1], and the Spanish flu is typically estimated to have caused between 17 and 50 million deaths worldwide from 1918 to 1920 [2] [3]. In the 21st century, major pandemics include SARS (Severe Acute Respiratory Syndrome) in 2002, Swine flu in 2009, MERS (Middle East Respiratory Syndrome) in 2015, and the most recent COVID-19 from late 2019. 
The first confirmed COVID-19 case in South Korea was recorded on 19 January 2020 - a Wuhan resident visiting South Korea [4]. The number of cases increased rapidly over the following months with over 13,550 confirmed cases, recorded as of 15 July 2020 [5]. The spread of the disease was exponential. In January, there were only 11 confirmed cases. By February, the number grew to 3150 and in March 9786. With government interventions and the deployment of location-based applications to enable social distancing, the curve began to flatten, with the number of cases increasing to 10,765 in April and then 11,468 in $\mathrm{May}^{1}$ [6].

The COVID-19 outbreak globally, is proving inherently difficult to manage because transmission can occur when people are pre-symptomatic or asymptomatic and not just when they have symptoms. This has meant that people may be unaware that they have the virus and are inadvertently spreading the disease [7]. The range of channels through which people can become infected has also made the spread of COVID-19 difficult to manage, with 'contact-based' transmissions caused by respiratory droplets, direct contact with contaminated objects and surfaces, and so forth [7].

To limit the spread of the disease and prevent community infections, the South Korean government launched a location-based service that uses mobile phone GPS technology to send notifications, via the Emergency Broadcasting Service, to people living in the vicinity of reported COVID-19 case/s. This service communicates the routes taken by COVID-19 patients and the places they visited. This official information is being released so that people will be able to go for COVID-19 screening in case they find overlapping pathways [8], and to permit people to avoid visiting affected areas if they are still being disinfected [6].

Private companies have also leveraged location-based health-related data to create more sophisticated visualisation applications using geospatial data so that people can see the contaminated areas and pathways of confirmed cases throughout the country. By taking prompt action with location-based applications and services, the government and private sector have jointly enabled South Korea to successfully flatten the curve - saving many lives and increasing community resiliency against COVID-19 in the longer term.

Between 30 January to 18 March 2020, several location-based applications were developed; each application playing a vital (but different) role in managing COVID-19 transmissions and needs - from tracking and tracing COVID-19 cases to locating pharmacies with available mask stocks, and so on. This chapter describes seven applications that have played a substantial role in communicating essential services to citizens during the pandemic, and controlling and preventing community-spread infections. The applications are:

- Emergency Broadcasting Service (CBS, cellular broadcasting service): Emergency text messaging transmission service that alerts people in the near vicinity of pathways taken by the COVID-19 infected people.

- Coronavirus Map: A website showing the location of all confirmed cases.

- Corona Now: A map-based information service providing information on national and international confirmed cases and mortalities, as well as real-time news broadcasts and the location of nearby COVID-19 screening centres, quarantined areas, and testing locations.

- Now and Here: Developed by ITL (Innovative Technology Lab), the 'Here and Now' App calculates a mix of risk factors and the percentage of risk associated with commuting routes based on identified COVID-19 cases.

- CoBaek Plus: Launched by a private developer Tina3D, CoBaek Plus App sends an alarm to a user when they are within 100 meters of a place that has had a confirmed case.

- Masks Stock App: An information system developed to resolve 'mask stock' shortages by showing the availability of face masks at various locations and recording purchases - where, when and by whom - to manage supply equitably.

\footnotetext{
${ }^{1}$ KCDC Statistics are accessible at https://github.com/jooeungen/coronaboard_kr/blob/master/kr_daily.csv
} 
- Self-Quarantine App: A self-monitoring and self-diagnosis service for citizens that is also used by government to monitor self-quarantined individuals when they are outside of designated quarantine areas.

There are numerous factors that contributed to the rapid development of COVID-19-related Apps, but the overreaching factors can be categorized into two major components:

\section{Systems}

\section{Policy/legal and institutional arrangements}

In terms of systems, the South Korean Government has a robust National Spatial Data Infrastructure (NSDI) in place. The NSDI provided the underpinning development environment for COVID-19 location-based applications. The NSDI consists of modern technologies, governance and policy frameworks, and wide-ranging fundamental and specialist data themes. The success of the NSDI is a result of a comprehensive and continuously updated NSDI Development Strategy that had guided enhancements to the NSDI over the past 20 years. The NSDI elements and policy/legal and institutional factors that have contributed to the success of the COVID-19-related applications and systems are described further in Section 24.4.

\subsection{Location-based Apps}

Location-based services are defined as 'services that integrate a mobile device's location (or position) with other information to provide added value to a user' [9]. Location-based applications (Apps) or websites, such as the Self-quarantine App, are created using these location-based services (Figure 24.1). Location data is used to pinpoint, visualise and integrate data, making it easier for people to understand the complex relationships between people, the economy and the environment.

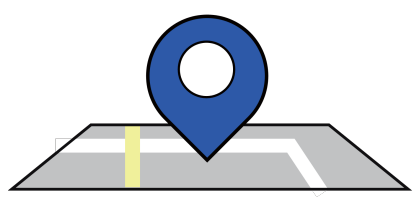

Location detection

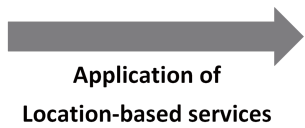

Location-based services

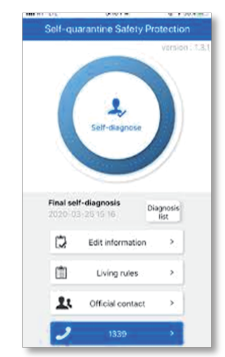

E.g. Self-quarantine App

FIGURE 24.1

Location-based services (LBS) and example of LBS App: Self-quarantine App

According to Seoul economy statistics, between February and April 2020, South Korea was ranked as having the highest growth rate $(135 \%)$ in monthly downloads of medical Apps during the COVID-19 pandemic. This contrasts with $65 \%$ growth rates, globally [10].

As COVID-19 spreads by personal contact, private companies and individuals quickly faced the challenge of developing location-based applications to show the locations of people with infections, enable social distancing, self-monitoring and self-diagnosis, and provide updates about new local cases, and the mitigation of face mask stockpiling. These applications, developed between January and March 2020, have played a significant role in saving lives and flattening the rate of infections.

The rapid development of COVID-19 applications was facilitated by several overarching 
factors including a high-level of science and technology education in both the developer- and user-community; a robust NSDI built on modern technologies, a solid data foundation and an open data policy; ubiquitous internet access and a high-number of mobile users able to access free smartphone Apps; and public trust in authorities and a 'collective' community culture.

The seven major Apps and websites' services, represented in Figure 24.2, are described below including their features, data sources and the location-based services used to control and prevent the spread of COVID-19 during the height of the pandemic.

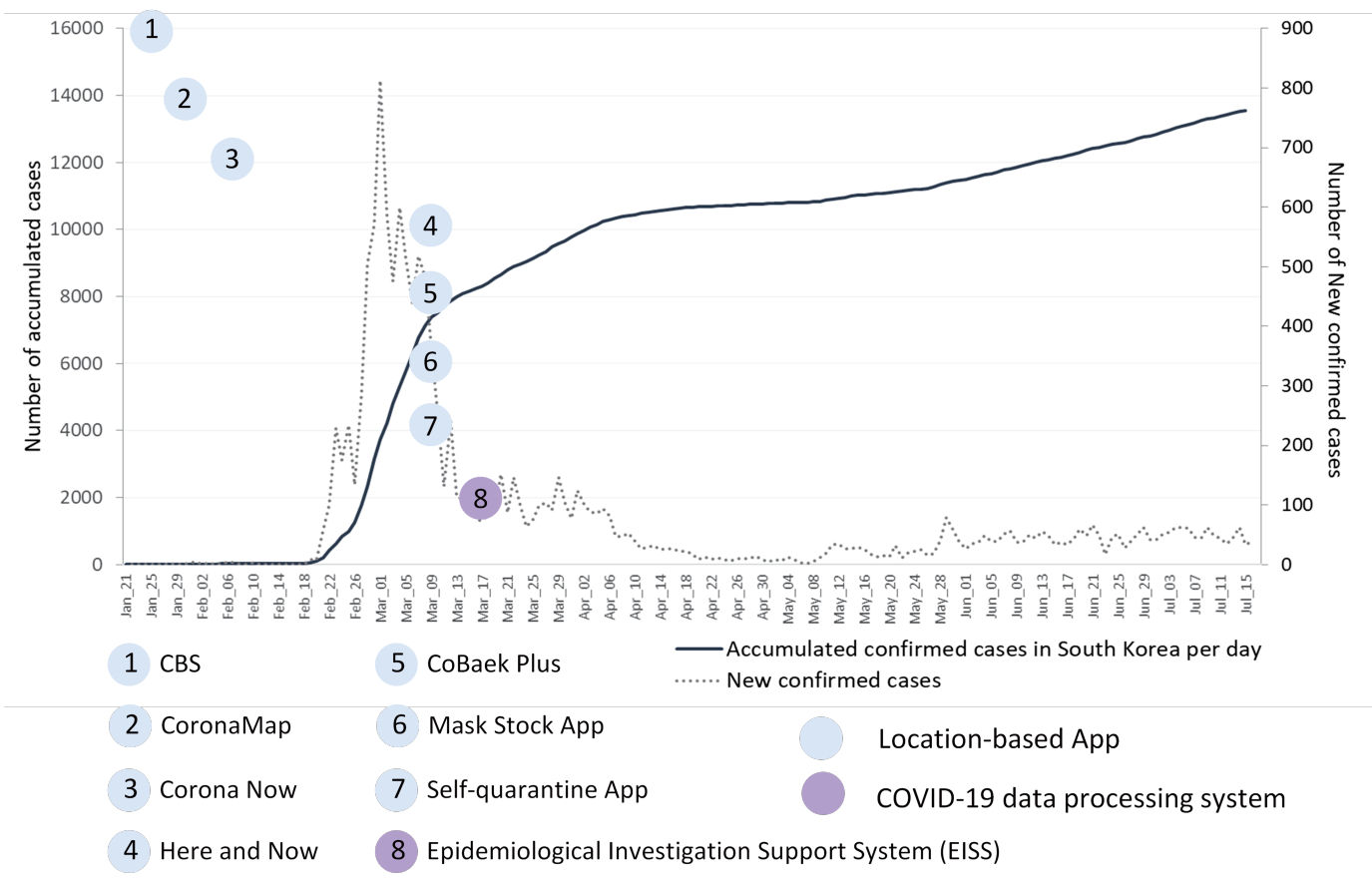

FIGURE 24.2

COVID-19-related location-based Apps in different timelines, and number of COVID-19 cases in South Korea; Statistics source: [5]

\subsubsection{Emergency Broadcasting Service}

The Emergency Broadcasting Service or CBS (Cellular Broadcasting Service) was first implemented for $2 \mathrm{G}$ cellular phones in 2005 by the Ministry of Interior \& Safety (MOIS). The service was developed to alert citizens to impending natural and manmade disasters in their local area so they could prepare early for potential eventualities [11], and to enable authorities to quickly respond to those natural disasters and avoid missing the 'golden time' [12], which occurs within the first hour following the onset of an emergency or disaster.

While the Ministry of Interior and Safety (MOIS) oversees the central CBS system and its operation, metropolitan and local governments can also send CBS messages to local residents and visitors in the vicinity of an emergency. The CBS service is an 'opt-in' subscriber service. Subscribers consent to having their mobile phone GPS location identified so that they can receive alerts. There is also an option to receive translated messages in English and Chinese.

In an endeavour to curb the contagion, municipalities throughout South Korea have been using Code 3 alerts to notify subscribers in the vicinity of COVID-19 infectious people.

The CBS uses COVID-19 confirmed patient 'pathway' data made available by the Korea Centers 
for Disease Control \& Prevention (KCDC). More specific information on COVID-19 patients' pathways is provided by local government. In South Korea, the data about COVID-19 patients is considered public data (i.e. the data collected from confirmed patients).

In addition to sending CBS alert messages to people living in the nearby vicinity of a patient, pathway tracing data is also uploaded to the MOIS CBS official website. In this way, all citizens can access the pathway data and, in turn, make informed decisions about social distancing and personal safety while commuting.

South Korean Laws on managing and openly sharing information on patients with infectious disease were enforced after the MERS outbreak in 2015 [8]. At the time of the MERS outbreak, South Korea ranked second for MERS confirmed cases, and the country was criticized for not having released patient location data publicly. This led the government to bring in new laws to enforce the sharing of information on the location of patients with infectious diseases.

However, while CBS has brought about far-reaching beneficial outcomes in the prevention of secondary infections, there have been criticism over privacy issues. Even though patients are not identified outright (i.e. by their name and address, which is protected data), citizens still dread stigma, fear losing their jobs or experiencing other potential hardship [13].

\subsubsection{CoronaMap}

The CoronaMap website enables people to make a visual inspection of where confirmed COVID-19 cases are using a map interface. This was an important design feature; while health officials release locations of COVID-19 patients, the official information is not very visual. CoronaMap not only pinpoints patient locations, it also connects the places they have visited with lines to show their pathways.

The data for CoronaMap is derived from the KCDC official website and managed by the CoronaMap database management team. An open API map from the $\mathrm{Naver}^{2}$ cloud platform provides the base map detail. CoronaMap includes a function that can determine a user's location using their mobile phone GPS. This enables them to compare their location with COVID-19 cases on the map and make an informed decision to go for COVID-19 screening because they have overlapping pathways (Figure 24.3).

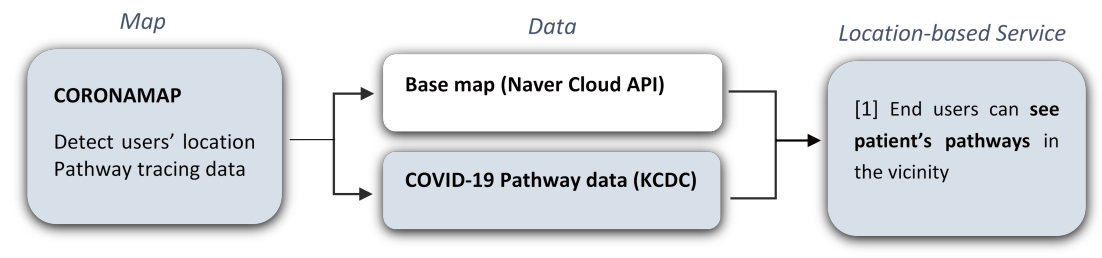

\section{FIGURE 24.3}

CoronaMap data components and service provided to end users

A link to CoronaMap was communicated through social media channels, such as Kakaotalk (a free mobile instant messaging application for smartphones) and Facebook. News of the application spread quickly, and the website had over 2.4 million viewers on the day it was launched [14]. According to in an article published by Hankyoreh Press, ${ }^{3}$ Dong-Hoon Lee expressed on his Facebook page 'The coding and the UI (User Interface) is still messed up. But I hope the website provides a useful service to the local community'. The public responded with messages of thanks and comments of gratitude for freely providing the CoronaMap website.

\footnotetext{
${ }^{2}$ NAVER Co., Ltd. is South Korea's largest web search engine, as well as a global ICT brand that provides services including LINE messenger, currently with over 200 million users from around the world, the SNOW video app, and the digital comics platform NAVER WEBTOON. Retrieved from: https://www. navercorp. com/en/naver/ company

${ }^{3}$ Hankyoreh Press is accessible at https://www.w3newspapers.com/south-korea/
} 


\subsubsection{Corona Now}

Corona Now was initially developed to provide COVID-19 national and international statistics dashboard and to fill an information delivery gap [15]. At the time, KCDC statistics on confirmed patients' pathways were only available via CBS messaging and there was a lack of information delivery on national and international dashboard in which people could see confirmed cases, number of discharged patients, or number of deaths at a glance. National statistics were only available at the country-level, and people wanted to have more accurate data at the district, province and neighbourhood levels, and be able to compare statistics at a global level. In addition to the statistics dashboard, Corona Now has added a location-based information service including the 'find the closest screening centre' feature.

Since its development, the community have used Corona Now extensively. Like CoronaMap, the Corona Now website link was communicated via social media. The Website was visited over 30,000 times in the first few days it was released. By 25 February, the total number of web visitors reached 2 million and was ranked first in the 'Naver Search' [16].

A key success of Corona Now stems from the fact that it draws information from reliable sources. Having access to authoritative data from the Korea Centres for Disease Control \& Prevention (KCDC), Johns Hopkins University Centre for Systems Science and Engineering (CSSE), and the China Medical information website ${ }^{4}[17]$ has meant that developers could analyse and process the data with confidence (Figure 24.4). This, in turn, has led to a high-level of community trust in location-based services.

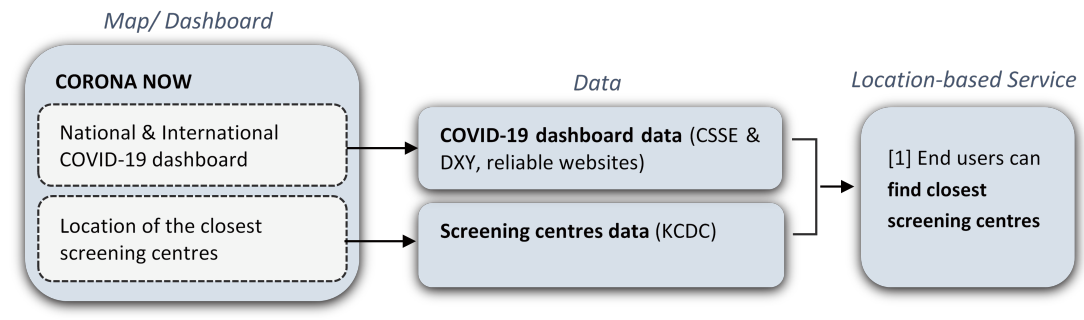

\section{FIGURE 24.4}

Corona Now data source, features and location-based service

\subsubsection{Now and Here}

The 'Now and Here' App is a community-based information sharing platform developed by private company Innovative Technology Lab (ITL). On 3 March 2020, ITL launched a new COVID-19 preventive service on the 'Here and Now' platform. The new feature calculates a mix of risk factors by detecting users' location and nearby infected areas. It also shows the percentage of 'risk' associated with commuting routes based on identified COVID-19 cases [18].

The Now and Here App not only shows the relative locations of the users to recent patient pathways in the vicinity, but also shows the closet COVID-19 screening centres, nearby ShinCheonJi churches, and recent news posts on COVID-19 (Figure 24.5). In March 2020, the mass outbreak of COVID-19 confirmed cases surged from a large gathering at the ShinCheonJi church in Daegu and spread the virus throughout the country. ShinCheonJi believers returned to their local ShinCheonJi churches and continued to spread the virus to the local community. Each local government hence decided to collect all ShinCheonJi Church locations, which were officially disclosed via the local government's official website (i.e. Seoul Metropolitan website, or Incheon city website), from which people could locate the church locations in their vicinity and avoid those areas [19]. The confirmed patients' data is sourced from KCDC, while news information is collected from reliable websites.

\footnotetext{
${ }^{4}$ China Medical information website is accessible at www.dxy.cn
} 


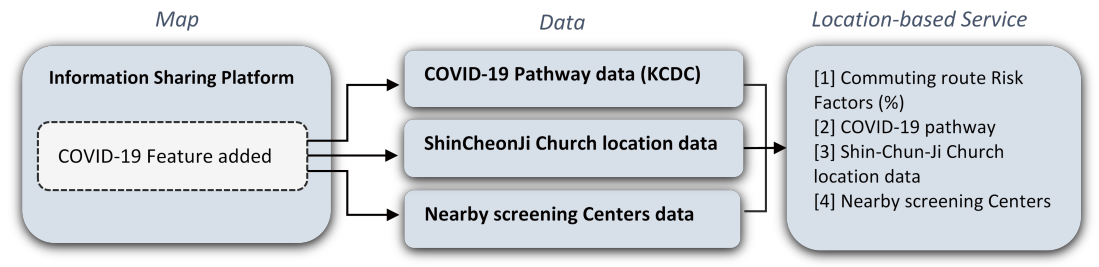

\section{FIGURE 24.5}

Here and Now data source and website services

The value of the new feature in the Now and Here App is that information is updated and refined as more information becomes available. For example, if a user posts a question in the App (e.g. where can I purchase face masks?); other users respond by uploading answers, relating to the user's region of interest within a radius of $0.5 \mathrm{~km}, 1 \mathrm{~km}, 2 \mathrm{~km}, 5 \mathrm{~km}, 10 \mathrm{~km}$ from the user's current location [20].

The Now and Here App has become one of the most essential Apps to have during the COVID-19 pandemic, particularly for commuters. In March 2020, the Now and Here App was ranked as one of the most downloaded Apps in Korea [21], because it provides diverse 'community-driven' location-based services that support both social-distancing measures and community well-being though knowing where essential services can be located [18].

\subsubsection{CoBaek Plus}

The 'Corona 100m' App was launched February 2020 and rereleased as 'CoBaek Plus' App on 18 March 2020 by private company Tina3D. ${ }^{5}$ Corona $100 \mathrm{~m}$ was first created to provide a visualisation of COVID-19 patient pathways, including the ability to send alarm messages to users, when they approach within a $100 \mathrm{~m}$ radius of a confirmed patients' recent pathways. In March 2020, the company updated 'Corona 100m' as 'CoBaek Plus' by adding new features such as Mask stock availability, and COVID-19 pathway tracing and withdrew the Corona 100m App.

'CoBaek Plus' has proven to be an ideal App for commuters and travellers who do not receive CBS messages nor regularly check COVID-19 pathway information. It is one of the most downloaded Apps in Korea. CoBaek Plus has achieved over 112,515 downloads and the earlier version Corona $100 \mathrm{~m}$ over 3,400,000 downloads as of February 26 [22]. One of the key success factors, is that the App is intuitive and easily downloaded by users. The developer, Tina3D, has not only received attention from the South Korean community but also from international communities and organizations. Mr. Bae, a board member of the company, said in an interview: 'We are currently in discussions with the Inter-American Development Bank (IDB) and the World Bank, Mexico and Spain' [22].

CoBaek Plus uses COVID-19 Pathway data released publicly by KCDC, and Mask Stock data is sourced from the government's open data platform. ${ }^{6}$ A map API (Naver) is used as the base map for the App and other location-based data, such as mask stock and pathway tracing data, are sourced from the Mask API (Figure 24.6) and pathway tracing API (from reliable sources such as Kaggle), respectively. National and International COVID-19 statistics data are sourced from KCDC for the national data, from WHO for international statistics and from BIDU for COVID-19 Statistics in China [22].

\footnotetext{
${ }^{5}$ TINA3D is accessible at http://www.tina3d.com/

${ }^{6}$ South Korea Open Data Platform is accessible at www.data.go.kr
} 


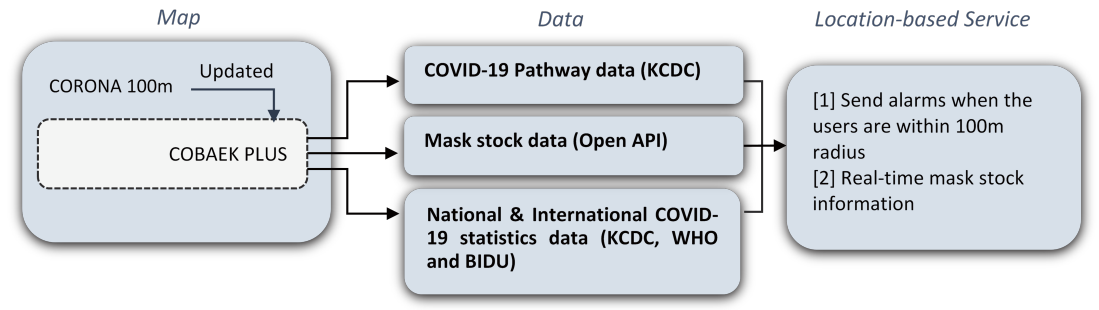

\section{FIGURE 24.6}

CoBaek Plus App data source and location-based services

\subsubsection{Masks Stock App}

Due to the scarcity of masks, the government launched the 'Five-day Rotation Face Masks' policy and distribution system on 9 March 2020. Under this policy, the general public can only purchase 2-3 face masks per week, and only on designated days. For instance, people with an ID number ending with 1 or 6 can only buy public masks on Monday, ID number ending with 2 or 7 on Tuesday, ID numbers ending with 3 or 8 on Thursday, and so on. Since people have only one day to buy public masks, checking on the mask stocks before heading to the nearby pharmacy was essential.

Masks are distributed to government-designated pharmacies, post offices and Nonghyup Hanaro Mart $^{7}$ stores. The sales of face masks are recorded by the government designated retailers, and then uploaded to the Health Insurance Review and Assessment Service (HIRA).

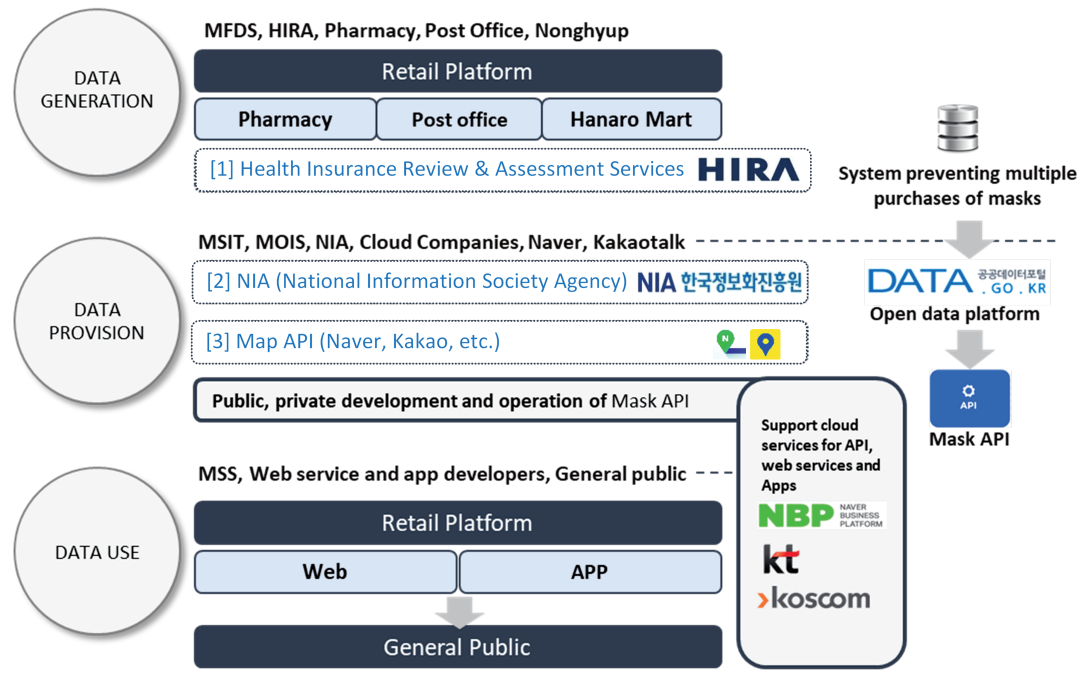

\section{FIGURE 24.7}

Publicly Distributed Face Mask Information Service

However, in order to manage the 'mask stock' shortage and direct the public to available stock, the government had to rapidly develop an information system showing the status of face mask stocks across the nation. To achieve this objective, the government took a Public-Private Partnership approach to development. In this partnership, the government released the data on face masks sold

\footnotetext{
${ }^{7}$ Name of a supermarket.
} 
at public designated-retailers through the public data centre of the National Information Society Agency (NIA). The NIA processed and published the HIRA data so it was accessible to the general public (as Open Data). Private companies could then develop cloud and other mapping services and Apps, through an Open API cloud service, provided free by the private sector (Figure 24.7).

The Public-Private Partnership approach was hugely successful. The face mask data was released by the government on 10 March 2020, and an App service was launched the very next day. The availability of Open Data through a sophisticated data infrastructure meant that it only took 13 hours to develop and deploy the first App service. Since then, more than 150 Apps have been developed to assist the public to purchase face masks. ${ }^{8}$ The number of data calls, related to mask distribution, through the API cloud reached 570 million (around 9.64 million per hour) from 11-31 March 2020 [12].

\subsubsection{Self-Quarantine}

The South Korean government developed the 'Self-quarantine Safety Application' to effectively support the monitoring of those under self-quarantine. The Android version was launched on 7 March and the iOS version on 14 March 2020. The Self-quarantine Safety Application supports three languages Korean, English and Chinese [12]. It comprises 3 main functions:

1. Self-diagnosis instructions for the users to conduct and submit results to an assigned government officer;

2. GPS-based location tracking to identify those outside their designated area; and

3. the provision of necessary health information including self-quarantine guidelines.

The App allows users to self-monitor their condition by uploading body temperature and answer survey questions relating to four symptoms: fever, cough, sore throat and respiratory difficulties (Figure 24.8). These survey results are automatically shared with their assigned officer. An alarm is triggered when a self-quarantine user does not submit their result. In addition, a monitoring officer gets an alarm when a person under self-quarantine violates self-quarantine restrictions (e.g. moving outside of the quarantine area). This information is retrieved from a person's mobile phone and submitted to designated officials.

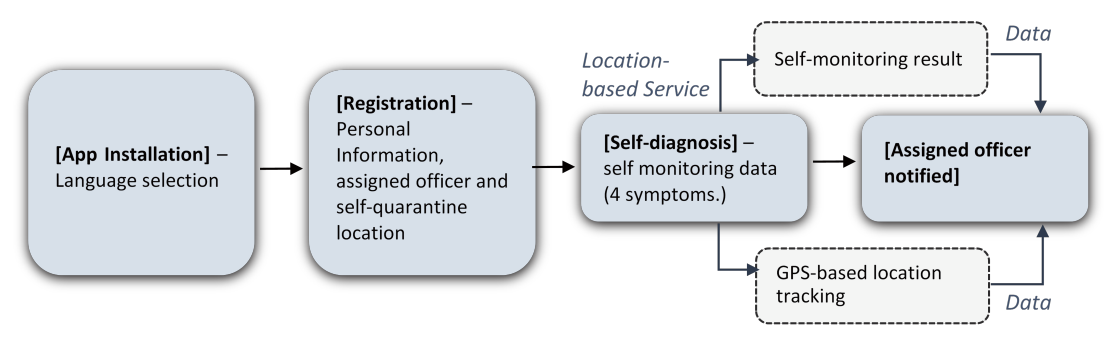

FIGURE 24.8

Process of Self-quarantine App use, and data generated

\subsection{Real-time Data Processing Systems}

Since 1954, the South Korean Government has been obligated to report all kinds of diseases, but omissions and delays were still apt to occur. As a consequence, the government decided to

\footnotetext{
${ }^{8}$ Apps for mark stock locations are accessible at http://mask.paas-ta.org
} 
take measures by introducing the 'Integrated Information Support System for Infectious Disease Control' in 2013 [23] to aggregate different disease-related reporting and management systems [24]. In 2015, the system was integrated with the 'Automatic Infectious Disease Report Support System', in which diagnosis results could be directly reported in real-time [24]. The integrated system has played a substantial role in controlling previous pandemics, MERS and SARS.

In 2015, the Infectious Disease Prevention and Control Act, originally enacted in 2009, was revised to enforce the disclosure of infectious disease patients and the collection and use of the data. This Act established a legal basis for the reporting, handling, securement, and disclosure of information required for epidemic investigations. Based on the Act, the Epidemic Investigation Support System (EISS) was developed as an application platform on the Smart City data platform [22].

\subsubsection{Integrated Information Support System for Infectious Disease Control}

The project to develop the 'Integrated Information Support System for Infectious Disease Control' was developed over a number of years and consisted of several development phases:

The Government was able to control the growth of infections for previous pandemics, MERS and SARS, solely by making use of these systems, mainly because the total confirmed cases and the rate of infections were fewer and with slower transmission rates than the recent COVID-19 pandemic. However, the Integrated Information Support System for Infectious Disease Control alone was not enough to manage and contain the spread of the virus, as COVID-19 is infected by contact, requiring more accurate location data.

\subsubsection{Smart City Data Hub}

The 'Smart City Data Hub' is a real-time platform that automatically collects city data using IoT devices (sensors and automatic reporting systems), which permit the analysis and visualisation of Big data without manual input, using technologies such as AI and machine learning. Data is collected across a wide range of sectors such as energy, transportation and city administration. The South Korean government launched the 'Smart City Innovation and Growth Project' at the national level in 2018. The project consists of three different focus areas:

1. Smart City Modelling;

2. Daegu Smart City Development Project; and

3. Si-Heung Smart City Development Project [25].

When the ShinCheonJi church-related COVID-19 outbreak occurred in March with hundreds of confirmed cases per day, the Ministry of Science and ICT, the Ministry of Land Infrastructure and Transport (MOLIT), KCDC, Police Department, Financial Service Committee, the Board of Audit and Inspection decided to launch the Epidemic Investigation Support System (EISS) into the 'City Data Hub' [26]. The EISS was therefore developed as an application built on the Smart City Data Hub [27] (Figure 24.9), that has been legally supported by the 'Infectious Disease Prevention and Control Act'. EISS contributed greatly to reducing time spent on analysing real-time analysis of large scale outbreak areas, including the visualisation of patients' pathways and hot spot locations. EISS also enabled App developers to use real-time data for other services. 


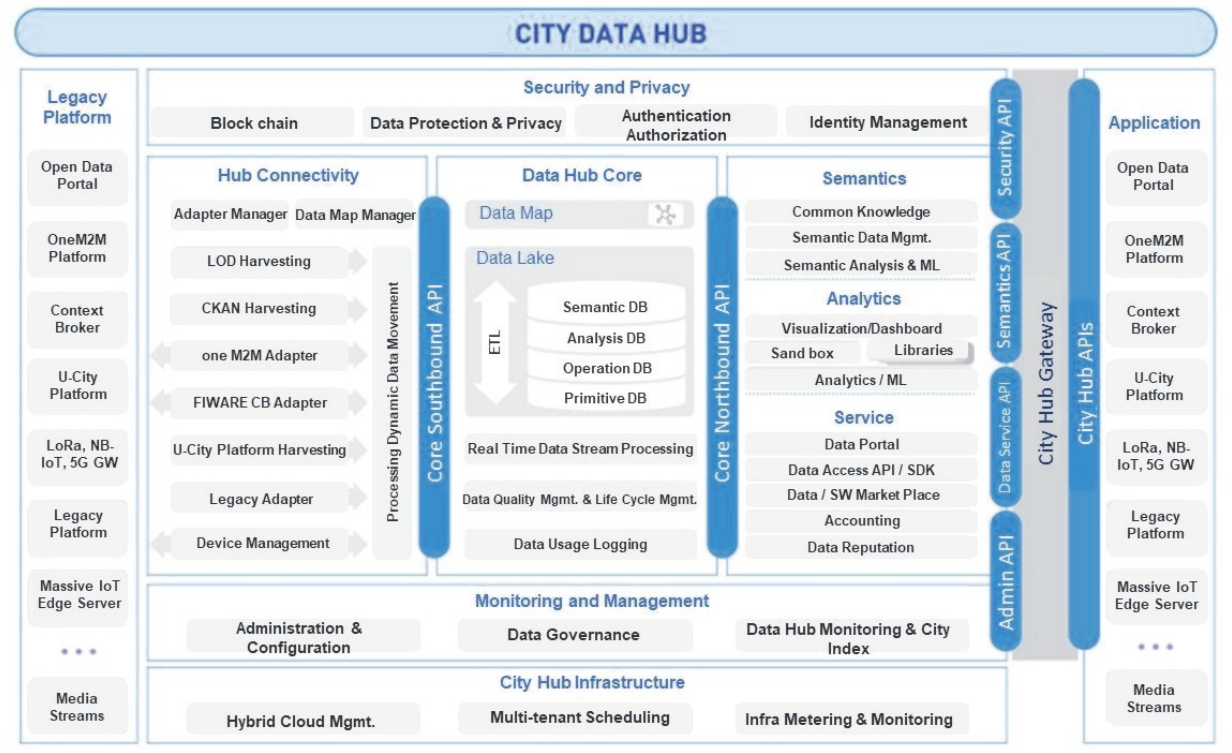

FIGURE 24.9

Structure of City Data Hub [27]

\subsubsection{Epidemiological Investigation Support System (EISS)}

According to the Infectious Disease Prevention and Control Act ${ }^{9}$ and its enforcement Decree, ${ }^{10}$ COVID-19-patient data are collected by the KCDC, under the Ministry of Health and Welfare (MOHW). The Act grants authority to the Police Department to ask for location information on infectious disease patient data that are normally retained by private mobile network companies. The Act also allows other responsible (designated) departments to collect additional data such as details of credit card usage (that has to be approved by the credit card association), visits to medical institutions, transport card use, entry and departure from the country, and closed-circuit television video footage.

In addition, the Infectious Disease Prevention Act has a provision to rapidly disclose the tracking information of infectious disease patients, depending on the outbreak situation [27]. The provision for the use of personal information amended in the Infectious Disease Prevention Act, including the disclosure of information for tracking purposes, was enforced during the MERS outbreak on 8 July 2015. To prevent the introduction and spread of dangerous infectious diseases, the Act was amended to enable the use of specific measures to rapidly control infectious diseases in the early stage of inflow [27].

According to the Infectious Disease Prevention Act, EISS has two different approval processes:

1. Location data: the Police Department has the authority to directly ask telecommunication companies (3 main national telecoms companies) to provide the requested data; and

2. Credit card-related data: the approval must be granted by the credit card association (22 credit card companies).

Following the EISS pilot operations conducted 16 March 2020, the system was officially launched on 26 March 2020 by the Ministry of Land, Infrastructure, and Transport (MOLIT), together with

\footnotetext{
${ }^{9}$ Infectious Disease Prevention and Control Act is accessible at http://elaw.klri.re.kr/eng_mobile/viewer.do? hseq $=53530 \&$ type $=$ part\&key $=36$

${ }^{10}$ The Decree is accessible at http://elaw.klri.re.kr/eng_mobile/viewer.do?hseq=43547\&type=part\&key=36
} 
the Ministry of ICT and Korea Centres for Disease Control and Prevention (KCDC) [26]. The EISS is operated in collaboration with private companies that provide location and card use data.

\subsection{COVID-19 Response Success Factors}

There are a number of factors that have contributed to South Korea's success in managing the COVID-19 pandemic. These factors include having a robust National Spatial Data Infrastructure (NSDI) that has provided the foundation for:

1. integrated geospatial data management and technological innovation;

2. an Open Data Policy that has stimulated the development of COVID-19-related systems and applications by government, private sector and individuals in the community;

3. institutional arrangements that have fostered a strong culture of data sharing;

4. high-quality fundamental and specific datasets; and

5. application development.

\subsubsection{National Spatial Data Infrastructure}

South Korea is recognized as one of the most influential digital economies powered by a well-coordinated NSDI [28], supportive policies and leadership. The NSDI success is founded on well-established and integrated geospatial datasets, interlinked Ministry and regional systems, a central database system, accessible information portals and cloud-based services that support application development.

It is the development of geospatial information-related technologies that has played a key role in enabling COVID-19 location-based products and services to be developed rapidly. In line with Industry 4.0, the geospatial information sector has experienced rapid innovation in parallel with major national agendas, such as Smart Cities and Ubiquitous Cities [29].

South Korea has continually developed and revised its overarching NSDI Basic Plan since 1995, and several major NSDI projects have been completed. The current 6th NSDI Basic Plan (2018-2022) aims to:

1. encourage the use of geospatial information in a way that creates social and economic value;

2. implement geospatial platforms to share information innovatively;

3. advance the geospatial information industry to stimulate job creation, and

4. augment geospatial information management through a public-participatory environment to enhance data quality and usability.

The presence of a well-established NSDI framework, including the legal system, and standards has provided the groundwork for base maps as an Open API format, which is essential for the development of COVID-19 location-based applications.

\subsubsection{Open Data Policy}

The rapid development of fit-for-purpose COVID-19 location-based Apps has provided far-reaching benefits for local communities faced with social distancing and precautionary travel. However, debate has sparked on the disclosure of private data: The Open Data Policy and other Acts, such as the Infectious Disease Prevention Act, have made it possible to legally disclose COVID-19 
confirmed patient data. While the Government took precautionary measures when sending CBS messages during the onset of the pandemic, the number of confirmed cases was so low to start with, that people could identify patients from news and media reporting.

As a consequence, people were more concerned about being stigmatized through news reports than benefitting from the released data [13]. As the severity of COVID-19 grew and there was increased fear in the virus, people came to trust the government in the management of their personal data.

\subsubsection{Institutional Arrangements}

A robust NSDI governance model, underpinned by a central coordinating body (NSDI Committee) and supportive institutional networks, has been an influencing factor in the development of COVID-19 applications and services, which rely on effective collaboration and data sharing. In addition, South Korea has embraced public-private partnerships (PPP). In 2011-12, an NSDI project introduced a new collaboration and data sharing model between local governments, central government and the private sector. This collaboration has since become entrenched in the fabric of government.

In order to better promote geospatial information-related industries, the government established the Spatial Information Industry Promotion Institute (SPACE N), to support geospatial information-related industries, support the operation of 3D geospatial maps (vWorld), promote research and development, and provide consultation for start-ups and other private sector businesses [30]. SPACE N has encouraged the development of new and innovative technologies, datasets and platforms, which have since become vitalizing components of the geospatial data market in South Korea and enablers for COVID-19 applications.

\subsubsection{Fundamental and Specific Dataset}

Fundamental datasets are provided through the 'NSDI Portal' and 'V-World Portal'11 (comprising 3D geospatial data), launched and operated by MOLIT. The list of fundamental datasets is based on the South Korean 'National Framework Data', which is progressively being translated to the set of Fundamental Data Themes endorsed by Member States at the Seventh Session of United Nations Committee of Experts on Global Geospatial Information Management (UN-GGIM).

Fundamental datasets are distributed via data portals and made available in various formats so that companies and individuals have greater options when developing new application and services. Formats include shapefiles, GPS (Global Positioning Systems) data and LBS (Location Based Services), Real-time data and open APIs. It is this breadth and variety of available high-quality integrated data that has made it possible to develop COVID-19 applications and services so quickly.

\subsection{Conclusion}

Several factors have contributed to the rapid development of these COVID-19 applications and services. The Spatial Information Industry Promotion Institute (SPACE N), established under MOLIT in 2012, has been promoting business start-ups and capacity building through education programs since 2012. The Open data policy adopted in 2013 played a substantial role in releasing base map data needed for the development of location-based Apps, supported by the robust NSDI that contributed in integrating geospatial data into one coordinate system (including for Smart City Data Hub) and aggregate the data into real-time geospatial platform.

These factors are categorized as:

1. Factor 1: Systems, which consist of technical components such as automation supported

\footnotetext{
${ }^{11}$ http://map.vworld.kr/map/maps.do
} 
by real-time systems that have contributed to processing COVID-19 mass data efficiently (discussed in Section 24.3); while

2. Factor 2: Policy/legal and institutional arrangements, including capacity development from national institutions such as SPACE N, which contributed to building highly skilled professionals in geospatial information and supported businesses and start-ups with enabling technologies and policy, which in turn had far- reaching impacts in procuring, releasing and sharing the base maps, which were essential to creating the location-based applications.

South Korea has been lauded for rapidly controlling and flattening the COVID-19 curve, but the location-based services and release of personal data has raised concerns with the public as they are used to trace and track people's movements, and are thus an invasion of privacy. These concerns remain unanswered, and the balance between public good and privacy is still debated.

In summary, hundreds of COVID-19 Apps were developed between the first outbreak up until to today (September 2020). However, privacy issues remain a key concern. Some Apps demand access to users' location and to other mobile device features, such as camera, to enable the collection of individuals private data. Legal enforcement, bridging the gap between public good and privacy, needs to be thoroughly addressed. In addition, with so many Apps available, there is need for a platform with a regularly updated list of COVID-19 Apps to enable the avoidance of similar services. This platform is expected to increase the efficiency and effectiveness of App development, enabling private developers to rapidly bridge communities' need in times of a pandemic.

What is clear from the Korean experience is that location-based Apps, in the hand of citizens, have delivered huge benefits in reducing the spread of COVID-19 because people have been able to make informed decisions about social distancing, their welfare and the welfare of others.

\section{References}

[1] J. Ole. The black death: The greatest catastrophe ever. History Today, 55(3), 2005.

[2] A. Trilla, G. Trilla, and C. Daer. The 1918 'spanish flu' in spain. Clinical Infectious Diseases, 47(5):668-673, 2008.

[3] CDC. 1918 pandemic (h1n1 virus), centre for disease control and prevention, 2019. URL https://www.cdc. gov/flu/pandemic-resources/1918-pandemic-h1n1.html.

[4] S. Koh. The first covid-19 confirmed case in south korea, 2020. URL https://www.doctorsnews.co.kr/news/ articleView.html?idxno=133009.

[5] Korea Centres for Disease Control and Prevention (KCDC). Statistics of covid-19 confirmed cases, 2020. URL http://ncov.mohw.go.kr/bdBoardList_Real.do.

[6] Korea Centres for Disease Control and Prevention (KCDC). Covid-19 statistics - regular briefing as of 21 may 2020, 2020. URL http://ncov.mohw.go.kr/tcmBoardView.do?contSeq=354638.

[7] World Health Organisation. Coronavirus disease 2019 (covid-19): situation report, 2020.

[8] BBC. Why is it the pathway tracing of the new covid-19 confirmed so detailed?, 2020. URL https://www. bbc.com/korean/news-51352575.

[9] J. Schiller and A. Voisard. Location-based services. Elsevier, 2004.

[10] J. Oh. Which country experienced rapid growth of medical applications during the height of covid-19 pandemic?, 2020. URL https://www.sedaily.com/NewsVIew/1Z44JU847J.

[11] MOIS. Sending sms (information service) for emergency disasters from the ministry of interior and safety (mois, 2017. URL https://www.gov.kr/portal/ntnadmNews/1219011.

[12] Republic of Korea. Flattening the curve on covid-19: How korea responded to a pandemic using ict, 2020.

[13] BBC. Coronavirus privacy: Are south korea's alerts too revealing?, 2020. URL https://www.bbc.com/news/ world-asia-51733145.

[14] J. Gang. Distribution of covid-19 confirmed cases at a glance, 2020. URL http://www.hani.co.kr/arti/ society/society_general/926540.html. 
[15] J. Moon. 'coronanow' developed by two middle school students in daegu... profits used for masks donation, 2020. URL https://www.ytn.co.kr/_ln/0103_202002241510065315.

[16] M. Kim. 'coronanow' app made naver company to reach out to the two middle school developers, 2020. URL http://news.imaeil.com/Society/2020022615263206129.

[17] BBC. Coronanow, coronamap...when pandemics meet technology, 2020. URL https://www.bbc.com/korean/ news-51541390.

[18] H. Lee. Covid-19 feature added in 'now and here' app, a community-based information sharing platform, 2020. URL https://m.etnews.com/20200303000313.

[19] P. Rosle. South korean church linked to many covid-19 cases is being sued for r1 billion in damages, 2020. URL https://www.businessinsider.co.za/south-korea-doomsday-church-shincheonji-sued-daegu-coronavirusdamages-2020-6.

[20] Maekyung. Now and here app, real-time information to market sales information, 2020. URL https://www. mk.co.kr/news/it/view/2018/08/532441/.

[21] B. Hwang. Masks and covid-19 pathway tracing... life with covid-19 became a routine, 2020. URL http: //www.dt.co.kr/contents.html?article_no=2020031802101958054004.

[22] J. Park. Tina3d receives love calls after developing coback plus app, 2020. URL http://www.newspim.com/ news/view/20200327000767.

[23] Korea Centres for Disease Control and Prevention (KCDC). Automatic infectious disease report support system, 2020. URL http://www.cdc.go.kr/npt/biz/npp/portal/nppAtRptMain.do.

[24] Korea Centres for Disease Control and Prevention (KCDC). Integrated information support system for infectious disease control, 2020. URL http://www.cdc.go.kr/contents.es?mid=a20301140000.

[25] Smart City Korea. Smart city innovation and growth r\&d, 2020. URL https://smartcity.go.kr/rd/.

[26] MOLIT. Covid-19 pathway tracing becomes faster and easier, 2020. URL http://m.molit.go.kr/viewer/skin/ doc.html?sfn=374f741a7cee499222a2ac204d1c2f 2d\&rs=/viewer/result/20200325.

[27] Y.J. Park, S.Y. Cho, J. Lee, O. Lee, W. Park, S. Jeong, S. Kim, S. Lee, J. Kim, and O. Parka. Development and utilization of a rapid and accurate epidemic investigation support system for covid-19. Osong Public Health and Research Perspectives, 11(3):118, 2020.

[28] E.H. Kim. A study on the strategic nsdi model for developing countries based on korean experiences. Spatial Information Research, 21(6):11-21, 2013.

[29] D.H. Shin. Ubiquitous city: Urban technologies, urban infrastructure and urban informatics. Journal of Information Science, 35(5):515-526, 2009.

[30] SPACE N. Introduction of space n, 2020. URL http://www.spacen.or.kr/spacen_intro/history.do. 
$\Longrightarrow$ Taylor \& Francis Taylor \& Francis Group

http://taylorandfrancis.com 